\title{
Identification of core genes and prediction of miRNAs associated with osteoporosis using a bioinformatics approach
}

\author{
YI CHAI ${ }^{*}$, FENG TAN* ${ }^{*}$ SUMIN YE, FEIXIANG LIU and QIAOLING FAN \\ Department of Formulaology of Traditional Chinese Medicine, School of Basic Medical Science, \\ Nanjing University of Chinese Medicine, Nanjing, Jiangsu 210046, P.R. China
}

Received January 31, 2018; Accepted August 23, 2018

DOI: $10.3892 / \mathrm{ol} .2018 .9508$

\begin{abstract}
Osteoporosis (OP) is an age-related disease, and osteoporotic fracture is one of the major causes of disability and mortality in elderly patients ( $>70$ years old). As the pathogenesis and molecular mechanism of OP remain unclear, the identification of disease biomarkers is important for guiding research and providing therapeutic targets. In the present study, core genes and microRNAs (miRNAs) associated with OP were identified. Differentially expressed genes (DEGs) between human mesenchymal stem cell specimens from normal osseous tissues and OP tissues were detected using the GEO2R tool of the Gene Expression Omnibus database and Morpheus. Network topological parameters were determined using NetworkAnalyzer. Gene Ontology (GO) and Kyoto Encyclopedia of Genes and Genomes pathway enrichment analyses were performed using the Database for Annotation, Visualization and Integrated Discovery, and ClueGO. Cytoscape with the Search Tool for the Retrieval of Interacting Genes and Molecular Complex Detection plug-in was used to visualize protein-protein interactions (PPIs). Additionally, miRNA-gene regulatory modules were predicted using CyTargetLinker in order to guide future research. In total, 915 DEGs were identified, including 774 upregulated and 141 downregulated genes. Enriched GO terms and pathways were determined, including 'nervous system development', 'regulation of molecular function', 'glutamatergic synapse pathway' and 'pathways in cancer'. The node degrees of DEGs followed power-law distributions. A PPI network with 541 nodes and 1,431 edges was obtained. Overall, 3 important modules were identified from the PPI network. The following
\end{abstract}

Correspondence to: Dr Qiaoling Fan, Department of Formulaology of Traditional Chinese Medicine, School of Basic Medical Science, Nanjing University of Chinese Medicine, 138 Xianlin Road, Nanjing, Jiangsu 210046, P.R. China

E-mail: njfanql@163.com

*Contributed equally

Key words: osteoporosis, bioinformatics analysis, topological parameter, protein-protein interaction, core gene, microRNA
10 genes were identified as core genes based on high degrees of connectivity: Albumin, $\mathrm{PH}$ domain leucine-rich repeat-containing protein phosphatase 2 (PHLPP2), DNA topoisomerase 2- $\alpha$, kininogen 1 (KNG1), interleukin 2 (IL2), leucine-rich repeats and guanylate kinase domain containing, phosphatidylinositol-4,5-bisphosphate 3-kinase catalytic subunit $\gamma(P I K 3 C G)$, leptin, transferrin and RNA polymerase II subunit A (POLR2A). Additionally, 15 miRNA-target interactions were obtained using CyTargetLinker. Overall, 7 miRNAs co-regulated IL2, 3 regulated $P H L P P 2,3$ regulated $K N G 1,1$ regulated $P I K 3 C G$ and 1 modulated $P O L R 2 A$. These results indicate potential biomarkers in the pathogenesis of OP and therapeutic targets.

\section{Introduction}

Osteoporosis (OP) is a major public health issue with a gradually increasing incidence, from $14.94 \%$ prior to 2008 to $27.96 \%$ in the period between 2012 and 2015 in China $(1,2)$. Nearly 27.5 million individuals in Europe were affected by OP in 2010 (3). Furthermore, the diagnosis of OP is difficult until the occurrence of bone fractures. Accordingly, research on the pathogenesis and molecular mechanism of OP is required in order to identify biomarkers and therapeutic targets.

Mutations in genes associated with OP have been detected. For example, cystatin A (expressed by the CSTA gene) is essential for epidermal development and maintenance (4). CSTA interacts with various genes, including one encoding a tyrosine kinase binding protein. These genes are associated with the immune regulation of osteoclasts (5). Additionally, a member of the fibroblast growth factors (FGFs) family, FGF21, is an essential endocrine hormone that regulates glucose and lipid metabolism (6). FGF21 affects bone development; it is inversely associated with regional bone mass density (BMD) (7). However, few studies have evaluated the core genes involved in OP using a bioinformatics approach. Although previous studies have identified several potential genes and proteins associated with OP, topological analyses are required in order to characterize the complex underlying networks. Furthermore, few studies have explored the microRNA (miRNA/miR)-gene regulatory networks to generate novel premises for OP research.

In the present study, the gene expression dataset GSE35956 was selected from the Gene Expression Omnibus (GEO) 
database (https://www.ncbi.nlm.nih.gov/geo) (8), and the GEO2R online analysis software (https://www.ncbi.nlm.nih. gov/geo/geo2r/) was used to uncover differentially expressed genes (DEGs). Using these loci, a protein-protein interaction (PPI) network was obtained and a network topological analysis was performed in order to identify core genes with high degrees of connectivity. In addition, the functions of the DEGs and 3 central modules were analyzed, including analyses of over-represented biological processes (BPs), molecular functions (MFs), cellular components (CCs), and Kyoto Encyclopedia of Genes and Genomes (KEGG) pathways $(9,10)$. miRNAs that regulate these core genes were predicted, providing a basis for further studies.

\section{Materials and methods}

Data. The gene expression dataset GSE35956 was obtained from the publicly accessible GEO database. GSE35956 was obtained through rigorous scientific design and the data can be analyzed with high quality. The selection criteria were as follows: i) Entry type: Series; ii) Organism: Homo sapiens; and iii) Experiment type: Expression profiling by array. The dataset includes human mesenchymal stem cell (hMSC) samples from 5 middle-aged donors without any indication of the syndrome (age range, 42-67 years; mean age, 57.6 years; sex, 4 female and 1 male) and 5 patients (age range, 79-94 years; mean age, 86.2 years; sex, female) suffering from primary OP (hMSC-OP) (11). Non-osteoporotic donors with total hip arthroplasty due to osteoarthritis and/or hip dysplasia were selected and MSCs from the bone marrow of the donors were obtained. MSCs of OP donors were isolated from femoral heads following low-energy fracture of the femoral neck. Benisch et al (11) used the Significance Analysis of Microarrays software (http://statweb.stanford.edu/ tibs/SAM/) to compare gene expression patterns of 2 groups of hMSC populations. In the present study, a separate analysis of 2 groups was performed and each group contained the 5 aforementioned samples. The study was based on the GPL570 platform (Affymetrix Human Genome U133 Plus 2.0 Array; Affymetrix; Thermo Fisher Scientific, Inc., Waltham, MA, USA). The series matrix file of GSE35956 was downloaded from the GEO database.

Data processing for DEG identification. GEO2R and Morpheus (https://software.broadinstitute.org/morpheus/) were used to identify DEGs among experimental samples (12). GEO2R offers a convenient interface enabling sophisticated R-based analyses of GEO data and is useful for the identification and evaluation of DEGs (8). $\mathrm{P}<0.05$ and $\mid \log \mathrm{FCl} \geq 2$ were set as the thresholds for DEG detection.

Gene Ontology (GO) and KEGG pathway analyses of DEGs. $\mathrm{GO}$ analyses are used to annotate genes or gene products and to determine biological characteristics of high-throughput genome or transcriptome data (10). KEGG is a group of databases for various biological data, including genomes and biological pathways. The Database for Annotation, Visualization and Integrated Discovery v6.8 (DAVID; https://david.ncifcrf.gov/) is a net-based online bioinformatics resource with tools for the functional interpretation of large-scale gene or protein datasets (13). The Functional Annotation Tool of DAVID was used and then the upregulated and downregulated DEGs were inserted into the tool for GO and KEGG analysis, respectively. In particular, Homo sapiens were required to be selected in order to limit the annotation of the species, thereby generating a summary of gene-species mapping. Finally, the data was downloaded and $\mathrm{P}<0.05$ was used to indicate a statistically significant difference.

Construction of functional annotation maps. In order to evaluate the biological functions of the proteins in the PPI networks based on upregulated, downregulated and total DEGs, the ClueGO plug-in v2.5.0 was used to identify over-represented BP terms for protein members in the network (14). ClueGO integrates GO terms into a PPI network and creates a functional annotation map that represents the associations between terms. The $\kappa$ score was set to 0.4 , indicating the resemblance of GO terms for associated genes. In addition, KEGG pathways were used to combine associated genes with corresponding pathways and $\mathrm{P}<0.05$ indicated a significant difference.

Network topological parameters. Network topological analyses were used for the comparison and characterization of complex networks. NetworkAnalyzer v2.7 (Department of Computational Biology and Applied Algorithmics at the Max Planck Institute for Informatics, Saarbrücken, Germany), which is part of Cytoscape v3.6.0, was used to analyze network topological parameters (15). NetworkAnalyzer only fits points with positive coordinate values and provides the association between the given data points and the corresponding points on the fitted curve. This coefficient provides the ratio of variability in a dataset, which is described by a fitted linear model. To determine whether two random variables satisfy the linear association, the correlation coefficient can be calculated with the linear regression model and the least squares method. Consequently, the $\mathrm{R}^{2}$ value is calculated on logarithmized data, where the power-law curve: $y=a x^{b}$ is transformed into linear model: $\ln \mathrm{y}=\ln \mathrm{a}+\mathrm{b} \ln \mathrm{x}$, where $\mathrm{x}$ and $\mathrm{y}$ are positive random variables, and $\mathrm{a}$ and $\mathrm{b}$ are constants $>0$. The power law distribution appears as a straight line with a slope of a power exponent. This linear association is the basis for judging whether a random variable satisfies a power law in a given instance, and the power-law distribution of node degree was the main parameter used to evaluate the network topology.

PPI network and module analysis. The Search Tool for the Retrieval of Interacting Genes (STRING) v10.5 (https://string-db. org/) is an online tool designed to evaluate PPI information (16) and this was used to detect potential associations among the DEGs. The results were input into Cytoscape v3.6.0 (http://www. cytoscape.org/) to map the associations among the DEGs (17). A confidence score $\geq 0.4$ and a maximum number of interactors of 0 were set as criteria. Molecular Complex Detection (MCODE) v1.5.1 (Bader Lab, University of Toronto, Toronto, Ontario, Canada) was used to evaluate the modules in the PPI network in Cytoscape (18). The definition of false discovery rate (FDR) is the expected value of the number of false rejects as a percentage of all rejected null hypotheses. The $\mathrm{P}$-value is generally performed using a Student's t-test or $\chi^{2}$ test for the analysis of differential gene expression, and the false positive test can be performed on the P-value using the Benjamini FDR check 
A

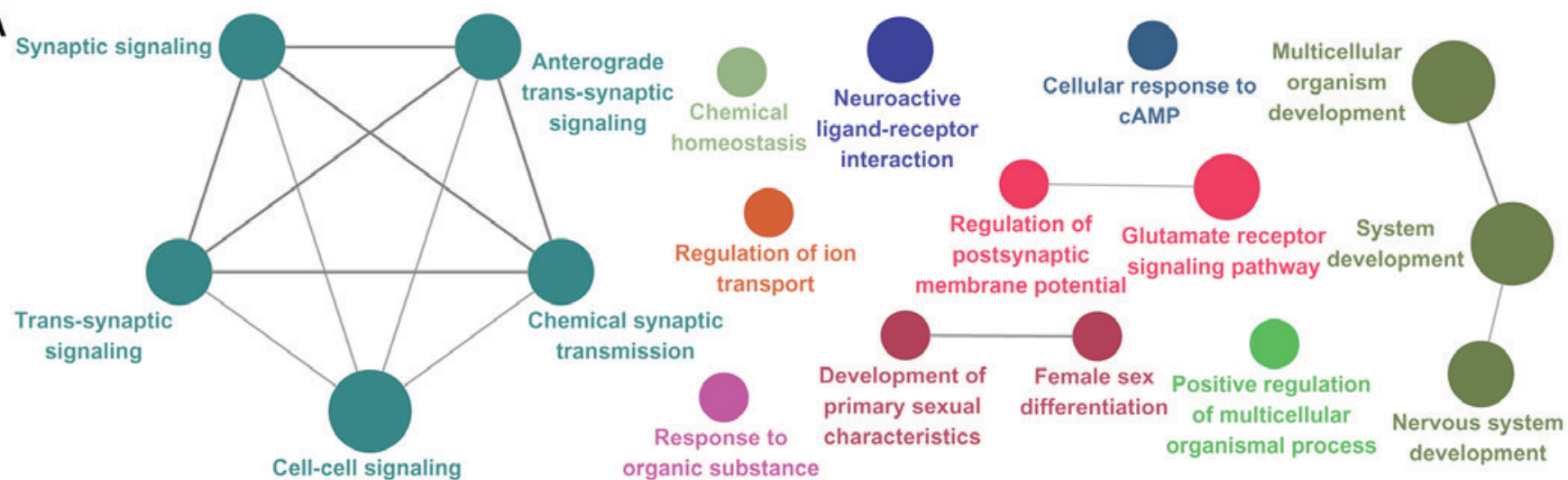

B

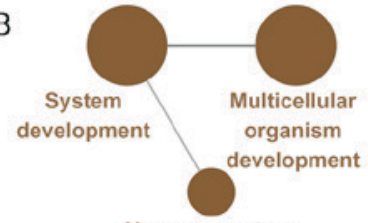

Nervous system

development Regulation of membrane potential Synaptic signaling

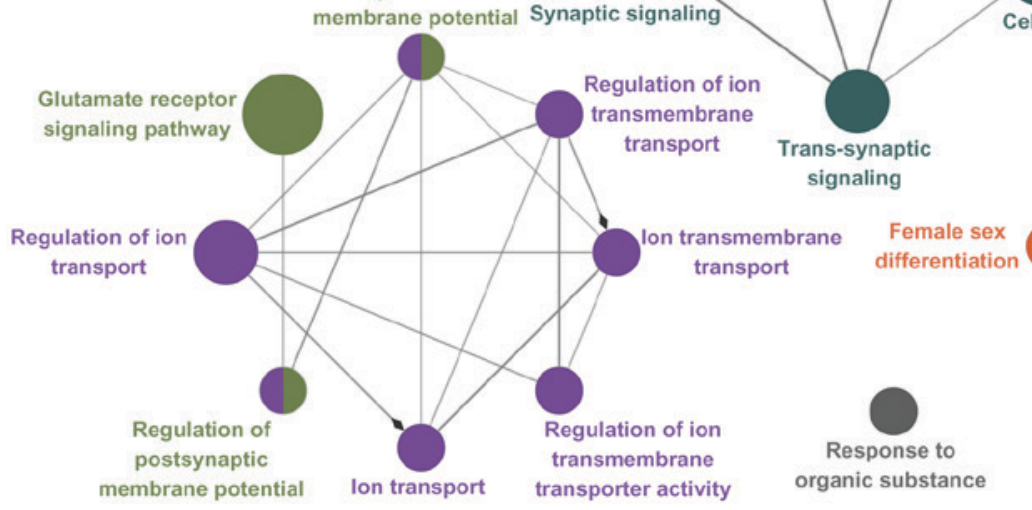

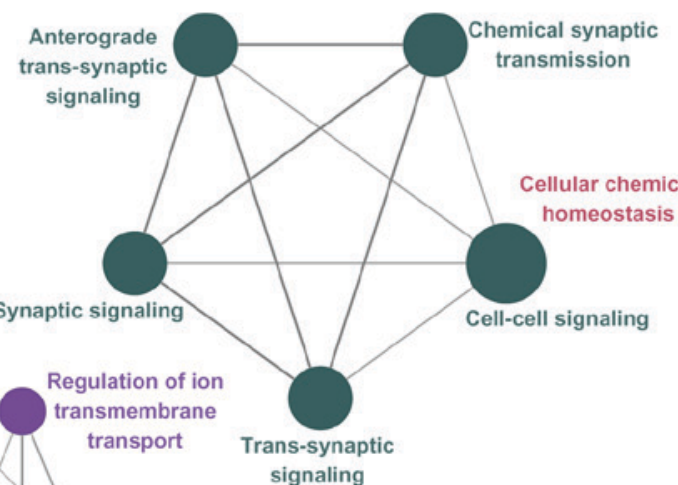

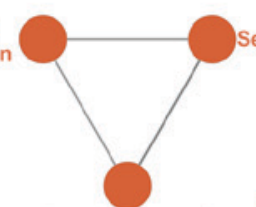

Development of primary sexual characteristics
Cellular response to
CAMP

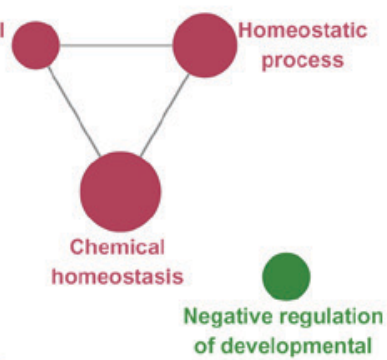

Sex differentiation process

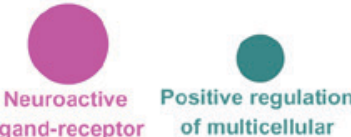

ligand-receptor of multicellular

interaction organismal process

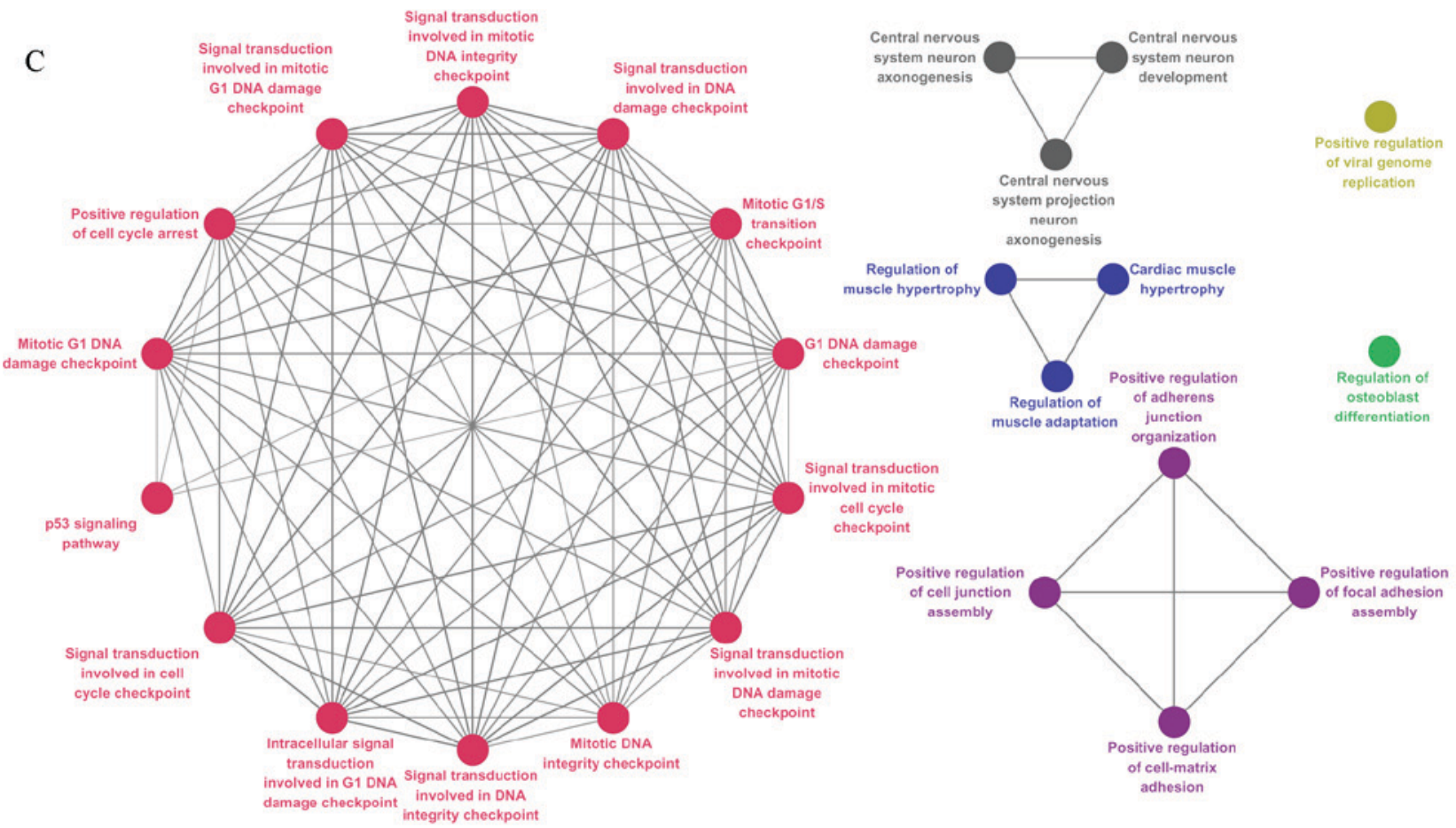

Figure 1. Functional annotation map of PPI subnetworks for (A) all DEGs, (B) upregulated DEGs and (C) downregulated DEGs. The GO biological process terms for total, upregulated and downregulated DEGs included in the PPI subnetworks are represented in (A), (B) and (C), respectively. GO terms are presented as nodes. PPI, protein-protein interactions; DEG, differentially expressed gene; GO, Gene Ontology. 
Table I. Gene Ontology analysis of differentially expressed genes associated with osteoporosis ${ }^{\mathrm{a}}$.

\begin{tabular}{|c|c|c|c|c|c|}
\hline Expression & Category & Term & Count $^{\mathrm{b}}$ & $\%^{\mathrm{c}}$ & P-value \\
\hline \multirow[t]{15}{*}{ Upregulated } & GOTERM_BP_FAT & GO:0007399 nervous system development & 106 & 13.73 & $4.20 \times 10^{-6}$ \\
\hline & GOTERM_BP_FAT & GO:0042592 homeostatic process & 88 & 11.40 & $1.06 \times 10^{-6}$ \\
\hline & GOTERM_BP_FAT & GO:0007267 cell-cell signaling & 85 & 11.01 & $2.52 \times 10^{-7}$ \\
\hline & GOTERM_BP_FAT & GO:0048878 chemical homeostasis & 66 & 8.55 & $1.42 \times 10^{-7}$ \\
\hline & GOTERM_BP_FAT & GO:0055082 cellular chemical homeostasis & 47 & 6.09 & $3.77 \times 10^{-6}$ \\
\hline & GOTERM_MF_FAT & $\begin{array}{l}\text { GO:0000981 RNA polymerase II transcription } \\
\text { factor activity, sequence-specific DNA binding }\end{array}$ & 43 & 5.57 & $1.96 \times 10^{-5}$ \\
\hline & GOTERM_MF_FAT & GO:0015267 channel activity & 31 & 4.02 & $3.65 \times 10^{-4}$ \\
\hline & GOTERM_MF_FAT & $\begin{array}{l}\text { GO:0022803 passive transmembrane transporter } \\
\text { activity }\end{array}$ & 31 & 4.02 & $3.80 \times 10^{-4}$ \\
\hline & GOTERM_MF_FAT & GO:0015276 ligand-gated ion channel activity & 16 & 2.07 & $6.49 \times 10^{-5}$ \\
\hline & GOTERM_MF_FAT & GO:0022834 ligand-gated channel activity & 16 & 2.07 & $6.49 \times 10^{-5}$ \\
\hline & GOTERM_CC_FAT & GO:0045202 synapse & 51 & 6.61 & $7.19 \times 10^{-7}$ \\
\hline & GOTERM_CC_FAT & GO:0044456 synapse part & 42 & 5.44 & $4.81 \times 10^{-6}$ \\
\hline & GOTERM_CC_FAT & GO:0097060 synaptic membrane & 29 & 3.76 & $6.53 \times 10^{-8}$ \\
\hline & GOTERM_CC_FAT & GO:0045211 postsynaptic membrane & 22 & 2.85 & $4.28 \times 10^{-6}$ \\
\hline & GOTERM_CC_FAT & GO:0060076 excitatory synapse & 20 & 2.59 & $1.79 \times 10^{-5}$ \\
\hline \multirow[t]{15}{*}{ Downregulated } & GOTERM_BP_FAT & GO:0065009 regulation of molecular function & 34 & 24.29 & 0.001 \\
\hline & GOTERM_BP_FAT & $\begin{array}{l}\text { GO:1902589 single-organism organelle } \\
\text { organization }\end{array}$ & 23 & 16.43 & 0.001 \\
\hline & GOTERM_BP_FAT & GO:0007010 cytoskeleton organization & 18 & 12.86 & 0.002 \\
\hline & GOTERM_BP_FAT & GO:0043087 regulation of GTPase activity & 14 & 10.00 & $9.63 \times 10^{-4}$ \\
\hline & GOTERM_BP_FAT & GO:0030036 actin cytoskeleton organization & 12 & 8.57 & 0.002 \\
\hline & GOTERM_MF_FAT & GO:0000166 nucleotide binding & 29 & 20.71 & 0.003 \\
\hline & GOTERM_MF_FAT & GO:1901265 nucleoside phosphate binding & 29 & 20.71 & 0.003 \\
\hline & GOTERM_MF_FAT & $\begin{array}{l}\text { GO:0035639 purine ribonucleoside } \\
\text { triphosphate binding }\end{array}$ & 24 & 17.14 & 0.004 \\
\hline & GOTERM_MF_FAT & GO:0032550 purine ribonucleoside binding & 24 & 17.14 & 0.004 \\
\hline & GOTERM_MF_FAT & $\begin{array}{l}\text { GO:0005201 extracellular matrix structural } \\
\text { constituent }\end{array}$ & 5 & 3.57 & 0.003 \\
\hline & GOTERM_CC_FAT & GO:0031012 extracellular matrix & 10 & 7.14 & 0.015 \\
\hline & GOTERM_CC_FAT & GO:0048471 perinuclear region of cytoplasm & 10 & 7.14 & 0.044 \\
\hline & GOTERM_CC_FAT & GO:0005578 proteinaceous extracellular matrix & 8 & 5.71 & 0.017 \\
\hline & GOTERM_CC_FAT & GO:0031252 cell leading edge & 7 & 5.00 & 0.046 \\
\hline & GOTERM_CC_FAT & GO:0001726 ruffle & 6 & 4.29 & 0.006 \\
\hline
\end{tabular}

${ }^{a}$ The top 5 terms were selected according to P-value. ${ }^{\mathrm{b}} \mathrm{Count}$ is the number of enriched genes for each term. GO, Gene Ontology; BP, biological process; MF, molecular function; $\mathrm{CC}$, cellular component. ${ }^{\mathrm{C}}$ Represents the percentage of genes contained in each term to the upregulated or downregulated genes.

method (19). In other words, the second screening of DEGs can be performed by the FDR significance parameter. The screening conditions were as follows: Degree, 2; node score, 0.2 ; k-core, 2; and maximum depth, 100. A KEGG pathway enrichment analysis for DEGs in the module was performed using the Functional Annotation Tool based on the DAVID for these cluster genes and annotation summary results are depicted as a Functional Annotation Chart in Fig. 1. The count threshold was set to 2 , which means the minimum number of genes for the corresponding term is 2 . Additionally, Homo sapiens were required to be selected to limit the annotation of the species. $\mathrm{P}<0.05$ indicated a significant difference.
Prediction of miRNA-gene regulatory modules. CyTargetLinker v3.0.1 (https://projects.bigcat.unimaas. $\mathrm{nl} /$ cytargetlinker/) was utilized to predict miRNA-target interactions (MTIs) for the genes, which were presented in a graphical manner using an extension of the network (20), with the network established by the core genes can be extended to be associated with miRNAs. A regulatory interaction network (RegIN) is a network containing regulatory interactions, often derived from online interaction databases (20). To generate a RegIN using CyTargetLinker in Cytoscape, Homo sapiens MTIs were obtained from the experimentally validated database miRTarBase v4.4, which includes 20,942 MTIs, 
Table II. Kyoto Encyclopedia of Genes and Genomes pathway analysis of DEGs associated with osteoporosis ${ }^{\mathrm{a}}$.

\begin{tabular}{|c|c|c|c|c|c|}
\hline Category of DEGs & Term & Count $^{\mathrm{b}}$ & $\%^{\mathrm{c}}$ & P-value & Genes \\
\hline \multirow[t]{5}{*}{ Upregulated } & $\begin{array}{l}\text { hsa04080: Neuroactive } \\
\text { ligand-receptor } \\
\text { interaction }\end{array}$ & 28 & 3.63 & $2.34 \times 10^{-7}$ & $\begin{array}{l}\text { AVPR2, MCHR1, GRIK2, NPY2R, GRIK3, GABRB1, } \\
\text { F2RL1, GRIK5, HCRTR1,LTB4R, CNR1, P2RY1, } \\
\text { CHRNA4, GRID1, GABRG3, GABRA2, PTGER3, } \\
\text { NPBWR2, LEP, P2RY10, ADRB1, GRM2, PRLR, } \\
\text { GRM8, CHRM1, P2RX2, GHSR, TSHR }\end{array}$ \\
\hline & $\begin{array}{l}\text { hsa04015:Rap1 } \\
\text { signaling pathway }\end{array}$ & 19 & 2.46 & $1.45 \times 10^{-4}$ & $\begin{array}{l}\text { PIK3CG, ADCY1, MAGI2, PGF, EFNA2, SIPA1L2, } \\
\text { KITLG, FGF23, RGS14, PRKCB,CNR1,P2RY1, } \\
\text { RASGRP2, CALML6, RAPGEF3, ANGPT2, } \\
\text { RAPGEF1, AKT2, ITGA2B }\end{array}$ \\
\hline & $\begin{array}{l}\text { hsa04024:cAMP } \\
\text { signaling pathway }\end{array}$ & 16 & 2.07 & 0.002 & $\begin{array}{l}\text { PIK3CG, HCN2, ADCY1, PTGER3, CFTR, CNGB1, } \\
C N G B 3, A D R B 1, C H R M 1, C A M K 2 D, C A L M L 6, \\
\text { RAPGEF3, GHSR, CAMK2A,TSHR, AKT2 }\end{array}$ \\
\hline & $\begin{array}{l}\text { hsa04724:Glutamatergic } \\
\text { synapse }\end{array}$ & 11 & 1.42 & 0.004 & $\begin{array}{l}\text { SLC17A8, SLC1A2, ADCY1, DLGAP1, GRM2, } \\
\text { GRM8, GRIK2, GRIK3, GRIK5, SHANK3, PRKCB }\end{array}$ \\
\hline & $\begin{array}{l}\text { hsa04261:Adrenergic } \\
\text { signaling in } \\
\text { cardiomyocytes }\end{array}$ & 11 & 1.42 & 0.020 & $\begin{array}{l}\text { PIK3CG, ADCY1, ADRB1, CACNG8, PPP1R1A, } \\
\text { CACNB1,CAMK2D,CALML6, RAPGEF3,CAMK2A, } \\
\text { AKT2 }\end{array}$ \\
\hline Downregulated & $\begin{array}{l}\text { hsa05200:Pathways } \\
\text { in cancer }\end{array}$ & 7 & 55.00 & 0.030 & $\begin{array}{l}\text { FGFR2, LAMA4, WNT4, HSP9OB1, APC2, PIAS2, } \\
\text { CDK2 }\end{array}$ \\
\hline
\end{tabular}

${ }^{a}$ The top 5 terms were selected for upregulated DEGs and one term was selected for downregulated DEGs according to P-values. ${ }^{\mathrm{b} C o u n t}$ is the number of enriched genes for each term. DEGs, differentially expressed genes. ${ }^{c}$ Represents the percentage of genes contained in each term to the upregulated or downregulated genes.

and from 2 predicted miRNA databases, MicroCosm v5.0, with 541,039 MTIs, and TargetScan v6.2, with 511,040 MTIs (http://projects.bigcat.unimaas.nl/cytargetlinker/regins). Each regulatory interaction comprises 2 nodes, a source (regulatory component) and a target biomolecule, connected by a single directed edge. In the present study, the top 10 hub genes were selected for the extension of the network using CyTargetLinker.

\section{Results}

Identification of DEGs. The gene expression profiles of 10 samples in the gene expression dataset GSE35956, including $5 \mathrm{OP}$ and 5 control samples, were analyzed. A total of 915 DEGs between the OP and control samples were identified using GEO2R and Morpheus, including 774 upregulated and 141 downregulated genes.

GO function and KEGG pathway enrichment analysis. GO and KEGG pathway enrichment analyses were performed using DAVID and the ClueGO plug-in in order to gain a comprehensive understanding of the functions of the DEGs. The results of the GO analysis indicated that upregulated and downregulated genes were enriched for various BP terms, which are listed in Table I. For upregulated DEGs these included 'nervous system development', 'homeostatic process', 'cell-cell signaling', 'chemical homeostasis' and 'cellular chemical homeostasis', and for downregulated DEGs they included 'regulation of MF', 'single-organism organelle organization', 'cytoskeleton organization', 'regulation of GTPase activity' and 'actin cytoskeleton organization'. In the MF category, the upregulated genes were enriched for 'RNA polymerase II transcription factor activity, sequence-specific DNA binding', 'channel activity', 'passive transmembrane transporter activity', 'ligand-gated ion channel activity' and 'ligand-gated channel activity', while the downregulated genes were enriched for 'nucleotide binding', 'nucleoside phosphate binding', 'purine ribonucleoside triphosphate binding', 'purine ribonucleoside binding' and 'extracellular matrix structural constituent'. A CC analysis further demonstrated that the upregulated genes were enriched for 'synapse', 'synapse part', 'synaptic membrane', 'postsynaptic membrane' and 'excitatory synapse', while the downregulated genes were enriched for 'extracellular matrix', 'perinuclear region of cytoplasm', 'proteinaceous extracellular matrix', 'cell leading edge' and 'ruffle'.

In addition, 6 KEGG pathways were identified, as listed in Table II, involving the 'glutamatergic synapse', 'adrenergic signaling in cardiomyocytes', 'neuroactive ligand-receptor interaction', 'Rap1 signaling pathway' and 'cAMP signaling pathway' for upregulated DEGs, and 'pathways in cancer' for downregulated DEGs.

The results of the ClueGO analysis demonstrated that upregulated and downregulated DEGs were particularly enriched for BP terms, including 'multicellular organism development', 'system development', 'response to organic substance', 'nervous system development' and 'cell-cell signaling' for upregulated DEGs, and 'regulation of osteoblast differentiation', 'mitotic DNA 
Table III. Summary of ClueGO analysis of DEGs for biological processes associated with osteoporosis ${ }^{\mathrm{a}}$.

DEG expression GO ID GO term Genes, $\mathrm{n}^{\mathrm{b}} \quad \%^{\mathrm{c}} \quad$ P-value $\quad$ Genes

\begin{tabular}{llllll}
\hline Upregulated & GO:0007275 & $\begin{array}{l}\text { Multicellular } \\
\text { organism }\end{array}$ & 220 & 4.14 & $5.03 \times 10^{-7}$
\end{tabular}

ADAMTS9, ADCY1, AKT2, ALG5, AMOT, ANGPT2, ANKRD54, ANPEP, APLP1, APOH, AQP1, ARHGAP26, ASB4, ASCL2, ASNS, ATCAY, ATN1, AVPR2, AXIN2, BCAN, BMP7, $B M P 8 B, B R S K 1, B R S K 2, C A M K 2 A$, CAMK2D, CBX2, CCK, CCR4, CD24, CD74, CHRM1, CNGB1, CNR1, CNTN4, COL10A1, CPB2, CUX2, CYP19A1, DACH1, DCHS2, DDC, DMBX1, DNAH11, DRAXIN, DYRK1B, EFNA2, EGR2, EMX1, EN2, EPHA4, ESRRB, EZH1, F2RL1, FEZF2, FGF23, FOXE1, FOXG1, FOXO1, FOXP2, FZD10, GABRB1, GAL, GHSR, GJA5, GJB6, GPM6B, GPRC5B, HDAC10, HES4, HID1, HIPK1, HMGA2, HNF4A, HOOK1, HPRT1, HSPB6, HTN3, IBSP, IKZF1, IKZF3, IL2, IRF4, ITGA2B, JMJD6, JPH1, KCNIP2, KITLG, KLHL17, KLHL32, KLKB1, KMT2B, KRT40, LEP, LFNG, LHX2, LHX6, LHX8, LPAL2, LRRC7, LSAMP, LST1, LTBP4, LYL1, MAB21L2, MAGI2, MARK4, MCF2, MEF2D, MEX3C, MINK1, MMP11, MOBP, MOV1OL1, MT1G, МYCВРАР, МYH7В, МYОЗВ, МYO7А, NDRG2, NEUROG2, NFATC4, NME8, NOBOX, NPAS1, NPPC, NPY2R, NROB1, NR2F6, NRP2, NRXN1, NTRK3, OBSL1, OLFM1, P2RX2, P2RY1, PAK3, PCDH15, PCDH8, PCDHGC5, PCSK6, PCYT1B, PECAM1, PGF, PHOSPHO1, PIK3CG, PKP2, PLCG2, POLB, PPARGC1A, PPARGC1B, PPDPF, PRKCB, PRLR, PSG1, PTPRB, RAB11FIP4, RAD21L1, RAPGEF1, RAPGEF3, RARRES2, RAX, RFX4, RGS14, RIMS1, RIPPLY2, SCEL, SCN3B, SCRT1, SDK2, SEMA4D, SEMA6B, SERPINA5, SETDB2, SEZ6L, SF1, SFTPD, SH3TC2, SHANK3, SHOX, SIAH3, SIN3A, SLC17A8, SLC1A2, SLC8A3, SMIM6, SOST, SOX10, SOX2, SOX8, SPATA19, ST8SIA2, STMN4, STOX1, TAF10, TBX3, TBX6, TCF21, TEF, TERT, TEX11, THPO, TIE1, TNFRSF11A, TP63, TRIB1, TRIM54, TRPC6, TRPM1, TSHR, TSSK1B, TTPA, TUB, UBE4B, WDR72, WT1, XIRP2, ZFP36L1, ZFP36L2, ZFP42 ADAMTS9, ADCY1, AKT2, AMOT, ANGPT2, ANKRD54, ANPEP, APLP1, APOH, AQP1, ARHGAP26, ASB4, ASCL2, ASNS, ATCAY, ATN1, AVPR2, AXIN2, BCAN, BMP7, BMP8B, BRSK1, BRSK2, CAMK2A, CAMK2D, CCK, CCR4, CD24, CD74, CHRM1, CNGB1, CNR1, CNTN4, COL10A1, CPB2, CUX2, CYP19A1, DCHS2, DMBX1, DNAH11, DRAXIN, 
Table III. Continued.

\begin{tabular}{lllll}
\hline DEG expression & GO ID & GO term & Genes, $n^{b}$ & $\%$
\end{tabular}

$\begin{array}{rllll}\text { GO:0010033 } & \begin{array}{l}\text { Response to } \\ \text { organic } \\ \text { substance }\end{array} & 138 \quad 4.34 \quad<0.001\end{array}$

DYRK1B, EFNA2, EGR2, EMX1, EN2, EPHA4, ESRRB, EZH1, F2RL1, FEZF2, FGF23, FOXE1, FOXG1, FOXO1, FOXP2, FZD10, GABRB1, GAL, GHSR, GJA5, GJB6, GPM6B, GPRC5B, HDAC10, HES4, HID1, HIPK1, HMGA2, HPRT1, HSPB6, HTN3, IBSP, IKZF 1, IKZF3, IL2, IRF4, ITGA2B, JMJD6, JPH1, KCNIP2, KITLG, KLHL17, KLHL32, KLKB1, KMT2B, KRT40, LEP, LFNG, LHX2, LHX6, LHX8, LPAL2, LRRC7, LSAMP, LST1, LYL1, MAB21L2, MAGI2, MARK4, MCF2, MEF2D, MEX3C, MINK1, MOBP, MTIG, MYH7B, MYO3B, MYO7A, NDRG2, NEUROG2, NFATC4, NOBOX, NPAS1, NPPC, NPY2R, NROB1, NR2F6, NRP2, NRXN1, NTRK3, OBSL1, OLFM1, P2RX2, P2RY1, PAK3, PCDH15, PCDH8, PCDHGC5, PCSK6, PCYT1B, PECAM1, PGF, PHOSPHO1, PIK3CG, PKP2, PLCG2, POLB, PPARGCIA, PPARGC1B, PPDPF, PRKCB, PRLR, PSG1, PTPRB, RABIIFIP4, RAD21L1, RAPGEF1, RAPGEF3, RARRES2, RAX, RFX4, RGS14, RIMS1, RIPPLY2, SCEL, SCN3B, SCRT1, SDK2, SEMA4D, SEMA6B, SERPINA5, SETDB2, SEZ6L, SF1, SFTPD, SH3TC2, SHANK3, SHOX, SIN3A, SLC17A8, SLC1A2, SLC8A3, SMIM6, SOX10, SOX2, SOX8, ST8SIA2, STMN4, STOX1, TAF10, TBX3, TBX6, TCF21, TERT, TEX11, THPO, TIE1, TNFRSF11A, TP63, TRIB1, TRPC6, TRPM1, TSHR, TTPA, TUB, UBE4B, WDR72, WT1, XIRP2, ZFP36L1, ZFP36L2, ZFP42 $A B C A 2, A B C C 2, A D C Y 1, A I M 2, A K T 2$, ANGPT2, APLP1, AQP1, AQP4, ASNS, ATP6VOA4, AVPR2, BMP7, BMP8B, BRSK2, $C A C N B 1, C A M K 2 A, C A M K 2 D, C C D C 3$, CCL23, CCR4, CD24, CD6, CD74, CFTR, CGN, CHRM1, CHRNA4, CNR1, CPB2, CRLF2, CSF2RA, CUX2, CXCL5, DBH, DDC, EGR2, ELANE, EPHA4, ESRRB, F2RL1, FABP4, FGF23, FOXO1, FOXP2, FZD10, GABRB1, GAL, GBP5, GHSR, GJB6, GPD1, GRIK5, HCN2, HCRTR1, HFE2, HID1, HIPK1, HNF4A, HPRT1, IBSP, IL2, IL22RA2, IL37, IRF4, ITIH4, KANK2, KHSRP, LATS2, LEP, LMO3, LPAL2, LRRC19, LRRC3, LTBP4, MAGI2, MAP2K7, MAPK4, MCF2, MEFV, $M T 1 G, M T H F R, M T S S I L, N F A T C 4, N P P C$, NROB1, NR2F6, NRP2, NRXN1, NTRK3, P2RX2, P2RY1, PAK3, PAQR9, PCSK6, PELO, PGF, PID1, PIK3CG, PLCG2, POLB, POLR2A, PPARGCIA, PPARGC1B, PRKCB, 
Table III. Continued.

DEG expression GO ID GO term Genes, $n^{b} \quad \%^{c} \quad$ P-value $\quad$ Genes

GO:0007399 $\begin{aligned} & \text { Nervous system } \\ & \text { development }\end{aligned} \quad \begin{array}{llll}109 & 4.61<0.001\end{array}$
development

GO:0007267 Cell-cell signaling

Downregulated GO:0045667 Regulation of osteoblast differentiation

GO:0044774 Mitotic DNA integrity checkpoint

$5 \quad 4.42 \quad 0.001$
PRLR, PSG1, RAPGEF1, RAPGEF3, RARRES2, RERG, RIPOR1, RNASE7, RNF31, RXRB,SIN3A, SLC11A1, SLC1A2, SLC8A3, SORBS1, SOST, SOX10, SOX2, SOX30, TAT, TCF21, TERT, THPO, TIE1, TNFRSF11A, TP63, TRIB1, TSHR, TUB, UBE4B, WT1, ZFP36L1, ZFP36L2

ADCY1, AKT2, APLP1, AQP1, ARHGAP26, ASCL2, ATCAY, ATN1, AVPR2, BCAN, BMP7, BRSK1, BRSK2, CAMK2A, CAMK2D, CCK, CCR4, CHRM1, CNGB1, CNR1, CNTN4, CUX2, DMBX1, DRAXIN, EFNA2, EGR2, EMX1, EN2, EPHA4, EZH1, FEZF2, FOXG1, FOXP2, FZD10, GABRB1, GAL, GHSR, GPM6B, GPRC5B, HDAC10, HES4, HIPK1, HPRT1, IL2, KCNIP2, KLHL17, LEP, LHX2, LHX6, LHX8, LRRC7, LSAMP, LST1, MAB21L2, MAGI2, MARK4, MCF2, MEF2D, MINK1, MOBP, MYO7A, NDRG2, NEUROG2, NFATC4, NPAS1, NPPC, NROB1, NR2F6, NRP2, NRXN1, NTRK3, OBSL1, OLFM1, P2RY1, PAK3, PCDH15, PCDH8, PCDHGC5, PPARGC1A, RAPGEF1, RAX, RFX4, RGS14, RIMS1, SCN3B, SCRT1, SDK2, SEMA4D, SEMA6B, SEZ6L, SH3TC2, SHANK3, SLC17A8, SLC1A2, SLC8A3, SOX10, SOX2, SOX8, ST8SIA2, STMN4, TBX3, TBX6, TERT, TP63, TRPC6, TRPM1, TSHR, UBE4B, ZFP36L1 ADRB1, AMER3, ANPEP, AQP1, AXIN2, BRSK1, BRSK2, CACNB1, CADPS, CAMK2A, CCL23, CD24, CFTR, CHRM1, CHRNA4, CNR1, CNTN4, CUX2, CXCL5, DBH, DLGAP1, DRAXIN, EFNA2, EGR2, EPHA4, FGF23, FOXO1, FZD10, GAL, GHSR, GJA5, GPRC5B, GRID1, GRIK2, GRIK3, GRIK5, GRM2, GRM8, HCN2, HCRTR1, HMGA2, HNF4A, IL2, JPH3, KCNIP2, LATS2, LEP, LTBP4, MAGI2, MCHR1, MINK1, MYCBPAP, NDRG2, NETO1, NFATC4, NPBWR2, NPPC, NPTX2, NPY2R, NRXN1, P2RX2, P2RY1, PANX2, PCDH15, PCDH8, PCDHGC5, PGF, PKP2, PLCG2, RAPGEF1, RAPGEF3, RASL10B, RGS14, RIMS1, SCEL, SCN3B, SHANK3, SHISA9, SIRPG, SLC1A2, SLC5A7, SLC8A3, SORCS3, SOST, SOX10, SOX2, STXBP5L, SYNPO, TBX3, TERT, TNFRSF11A, TP63, TSHR, UNC13C FGFR2, IL6ST, PIAS2, PRKD1, WNT4 $5 \quad 4.31 \quad 0.001 \quad C D K 2, C N O T 7, P L K 3, T O P 2 A, T P 73$ 
Table III. Continued.

\begin{tabular}{|c|c|c|c|c|c|c|}
\hline DEG expression & GO ID & GO term & Genes, $\mathrm{n}^{\mathrm{b}}$ & $\%^{c}$ & P-value & Genes \\
\hline & GO:0021954 & $\begin{array}{l}\text { Central } \\
\text { nervous } \\
\text { system } \\
\text { neuron } \\
\text { development }\end{array}$ & 4 & 5.41 & 0.001 & $A D A R B 1, F G F R 2, N F I B, S Z T 2$ \\
\hline & GO:0044819 & $\begin{array}{l}\text { Mitotic G1/S } \\
\text { transition } \\
\text { checkpoint }\end{array}$ & 4 & 5.19 & 0.002 & CDK2, CNOT7, PLK3, TP73 \\
\hline & GO:0031571 & $\begin{array}{l}\text { Mitotic G1 } \\
\text { DNA damage } \\
\text { checkpoint }\end{array}$ & 4 & 5.19 & 0.002 & CDK2, CNOT7, PLK3, TP73 \\
\hline
\end{tabular}

${ }^{a}$ The top 5 terms were selected according to P-value. ${ }^{b}$ Number of enriched genes for each term. GO, Gene Ontology; DEGs, differentially expressed genes; BP, biological process. ${ }^{c}$ Represents the percentage of genes contained in each term to the upregulated or downregulated genes.

Table IV. Topological parameters for the total, the upregulated and the downregulated PPI subnetworks.

\begin{tabular}{lccccc}
\hline PPI subnetwork & $y=a x^{\mathrm{b}}$ & $\mathrm{R}^{2}$ & $\begin{array}{c}\text { Correlation } \\
\text { coefficient }\end{array}$ & $\begin{array}{c}\text { Clustering } \\
\text { coefficient }\end{array}$ & $\begin{array}{c}\text { Network } \\
\text { centralization }\end{array}$ \\
\hline Total & $y=256.19 x^{-1.478}$ & 0.863 & 0.931 & 0.249 & 0.137 \\
Upregulated & $y=191.96 x^{-1.462}$ & 0.901 & 0.939 & 0.256 & 0.153 \\
Downregulated & $y=18.646 x^{-1.291}$ & 0.809 & 0.984 & 0.142 & 0.287 \\
\hline
\end{tabular}

$\mathrm{R}^{2}$ is a measure of the fit quality, with a range of 0-1 (a higher value indicates a better fit). The correlation between observations and the corresponding points on the fitted curve was determined. Clustering coefficient denotes an average of the clustering coefficients of all nodes in the network. Network centralization is a connectivity distribution index. Network density is the normalized value of the average number of neighbors. PPI, protein-protein interaction.

Table V. Enriched pathways for modules A-C ${ }^{a}$.

\begin{tabular}{|c|c|c|c|c|}
\hline Modules & Terms & P-value & FDR & Genes \\
\hline A & $\begin{array}{l}\text { Neuroactive ligand-receptor } \\
\text { interaction }\end{array}$ & $1.53 \times 10^{-6}$ & 0.001 & MCHR1, PTGER3, GRM2, GRM8, CNR1, NPY2R, NPBWR2 \\
\hline B & Ubiquitin mediated proteolysis & 0.021 & 2.096 & UBA6, HERCl \\
\hline $\mathrm{C}$ & $\begin{array}{l}\text { Neuroactive ligand-receptor } \\
\text { interaction }\end{array}$ & $3.94 \times 10^{-9}$ & $2.36 \times 10^{-6}$ & HCRTR1, P2RY10, LTB4R, CHRM1, P2RY1, F2RL1, GHSR \\
\hline
\end{tabular}

${ }^{a}$ One term was selected for each module according to P-value. FDR, false discovery rate.

integrity checkpoint', 'central nervous system neuron development', 'mitotic G1/S transition checkpoint' and 'mitotic G1 DNA damage checkpoint' for downregulated DEGs (Table III). KEGG pathways involved in 'neuroactive ligand-receptor interaction' and the 'p53 signaling pathway' for upregulated and downregulated DEGs, respectively, were identified.

Functional annotation map of PPI subnetworks. ClueGO provided a functional annotation map for PPI subnetworks, in which protein members are enriched corresponding to their GO terms and pathways (Fig. 1). Regulation of osteoblast differentiation was linked to 5 genes. Among these, FGF receptor 2, interleukin 6 signal transducer and Wnt family member 4 genes were also associated with a number of BP terms, including 'central nervous system projection neuron axonogenesis', 'cardiac muscle hypertrophy', 'positive regulation of focal adhesion assembly biological process' and 'pathways in cancer'.

Analyses of network topological properties. The node degrees for the total upregulated and downregulated DEGs generally 
obeyed power-law distributions, as demonstrated in Fig. 2 . Supplementary topological parameters, including the clustering coefficient, network centralization and network density, are listed in Table IV.

Core genes and modules in the PPI network. The top 10 core genes with the highest node degrees were determined using Cytoscape and the STRING database. These were albumin $(A L B), \mathrm{PH}$ domain leucine-rich repeat-containing protein phosphatase 2 (PHLPP2), DNA topoisomerase 2- $\alpha$ (TOP2A), kininogen $1(K N G 1)$, interleukin 2 (IL2), leucine-rich repeats and guanylate kinase domain containing (LRGUK), phosphatidylinositol-4,5-bisphosphate 3-kinase catalytic subunit $\gamma$ $(P I K 3 C G)$, leptin $(L E P)$, transferrin $(T F)$ and RNA polymerase II subunit A (POLR2A). Using an MCODE analysis, several significant modules were identified, including 541 nodes and 1431 edges. The 3 modules with the lowest P-values were selected to evaluate enriched pathways (Fig. 3 and Table V).

Identification of miRNA-gene regulatory modules. CyTargetLinker was used to predict miRNA-gene interactions for the 10 core genes. In total, 305 predicted MTIs were identified using the MicroCosm database and 277 predicted MTIs were identified using the TargetScan database, including 456 nodes and 582 edges. Additionally, setting a threshold functionality of 2, 15 MTIs were found from the MicroCosm and TargetScan databases. The genes and miRNAs are shown in Fig. 4. Specifically, 7 miRNAs that co-regulate IL2, 3 miRNAs that regulate $P H L P P 2,3$ miRNAs that regulate $K N G 1,1$ miRNA that regulates $P I K 3 C G$ and 1 miRNA that modulates POLR2A were detected (Table VI).

\section{Discussion}

OP has become a pervasive public health problem; it is characterized by decreased bone strength and an increased risk of fractures (21). The pathogenesis of OP involves an imbalance between osteoclast resorption and osteoblast bone formation. MSCs have multiple differentiation potential, and they can directly participate in bone formation by phenotypic differentiation into osteoblasts and indirectly via paracrine effects. During the process of aging, MSCs in bone marrow transform into other types of cells, including adipocytes and osteoblasts, leading to OP (22). Owing to the high prevalence and gravity of OP, understanding its pathogenic and molecular mechanisms is urgent for drug development and treatment. Microarray and high-throughput sequencing approaches have been used extensively to predict therapeutic targets for diseases, including OP. In the present study, the gene expression dataset GSE35956 was obtained from the GEO database to explore core genes and predict regulatory miRNAs involved in OP. Specifically, $5 \mathrm{hMSC}$ specimens from normal osseous tissues and $5 \mathrm{hMSC}$ specimens from OP samples were included and 915 DEGs were identified, including 774 upregulated and 141 downregulated genes.

Subsequently, network topological properties were analyzed to distinguish between the predicted network and a random network. A node degree approximating a power-law distribution is a standard characteristic of scale-free networks, and the PPI network in this study obeyed this rule $(23,24)$. The 3 subnetworks were true complex biological networks
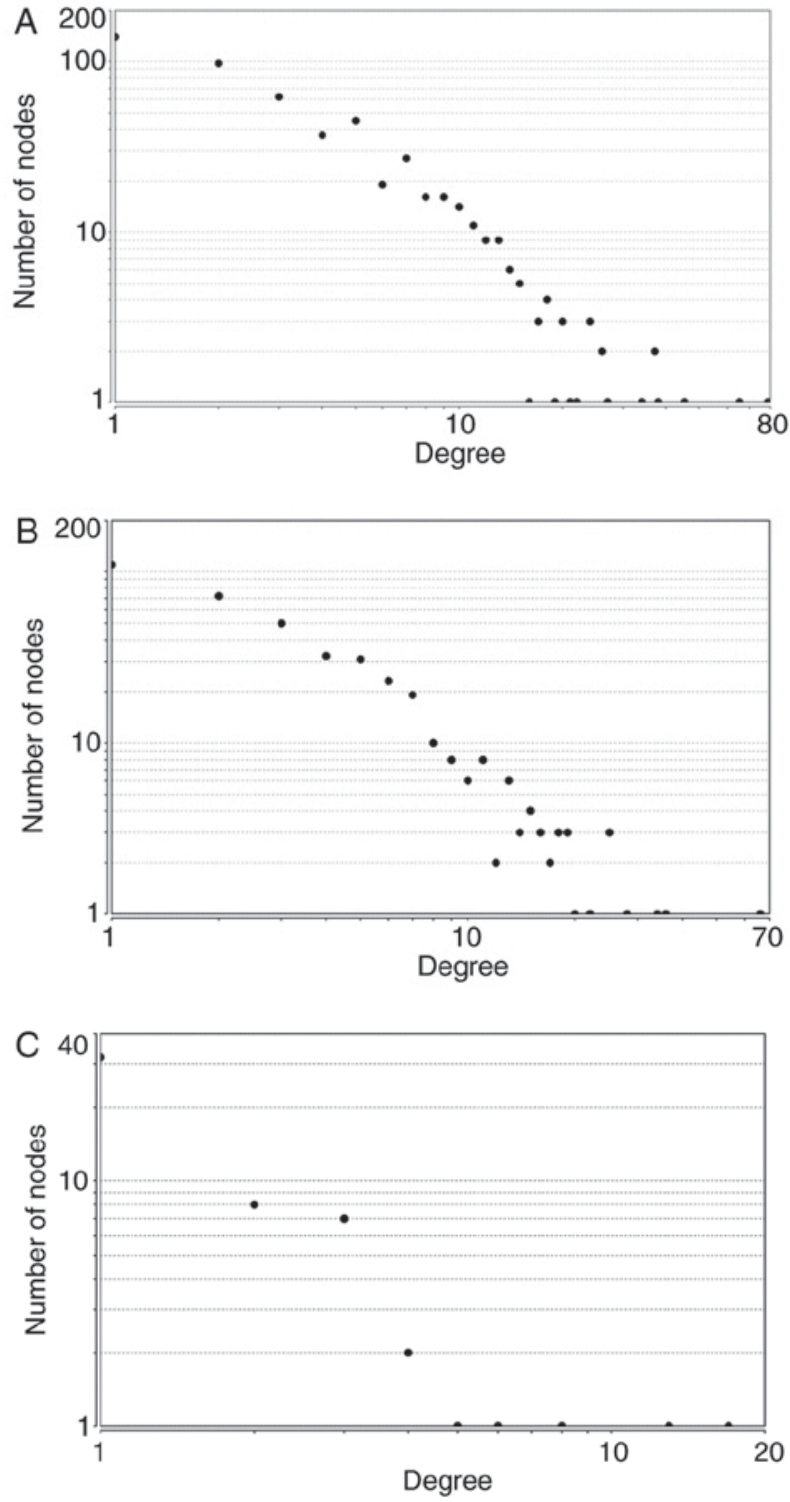

Figure 2. Power-law distributions of node degrees for (A) the total, (B) the upregulated and (C) the downregulated PPI subnetworks. Degree is a parameter used to describe the number of interacting partner proteins. As the number of linked nodes increases, the average degree decreases. PPI, protein-protein interactions.

characterized as scale-free, suggesting that the PPI network is reliable and robust.

OP-associated DEGs identified in the present study are not necessarily biologically meaningful. A number of effective methods, including GO and KEGG analyses, can be used to determine the importance of DEGs, with the goal of clarifying the roles of individual molecules in BPs. Accordingly, GO and KEGG pathway analyses were performed using the DEGs in the present study. Upregulated DEGs were primarily associated with 'nervous system development', 'multicellular organism development', 'RNA polymerase II transcription factor activity', 'sequence-specific DNA binding and synapse', while downregulated DEGs were associated with the 'regulation of molecular function', 'regulation of osteoblast differentiation', 'nucleotide binding' and 'extracellular matrix'. Based on the KEGG pathway analysis, upregulated DEGs were enriched for 'neuroactive ligand-receptor interaction', 'Rap1 signaling 
A
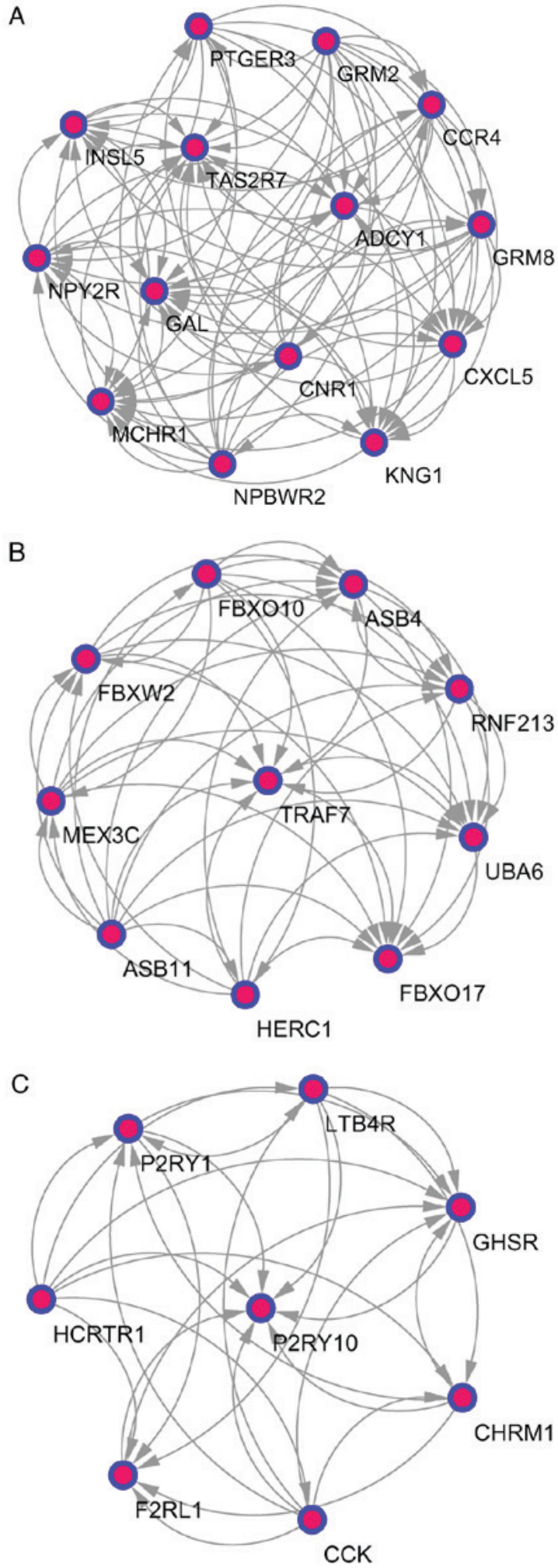

Figure 3. Top 3 modules from the protein-protein interactions network (A) Module A, 14 nodes and 91 edges; (B) module B, 10 nodes and 45 edges; and $(\mathrm{C})$ module $\mathrm{C}, 8$ nodes and 28 edges.

pathway', 'cAMP signaling pathway', 'glutamatergic synapse' and 'adrenergic signaling in cardiomyocytes', while the downregulated DEGs were involved in the 'p53 signaling pathway'.

Based on an analysis of the PPI network, the following core genes were identified: $A L B, P H L P P 2, T O P 2 A, K N G 1$, IL2, LRGUK, PIK3CG, LEP, TF and POLR2A. Among these,
A
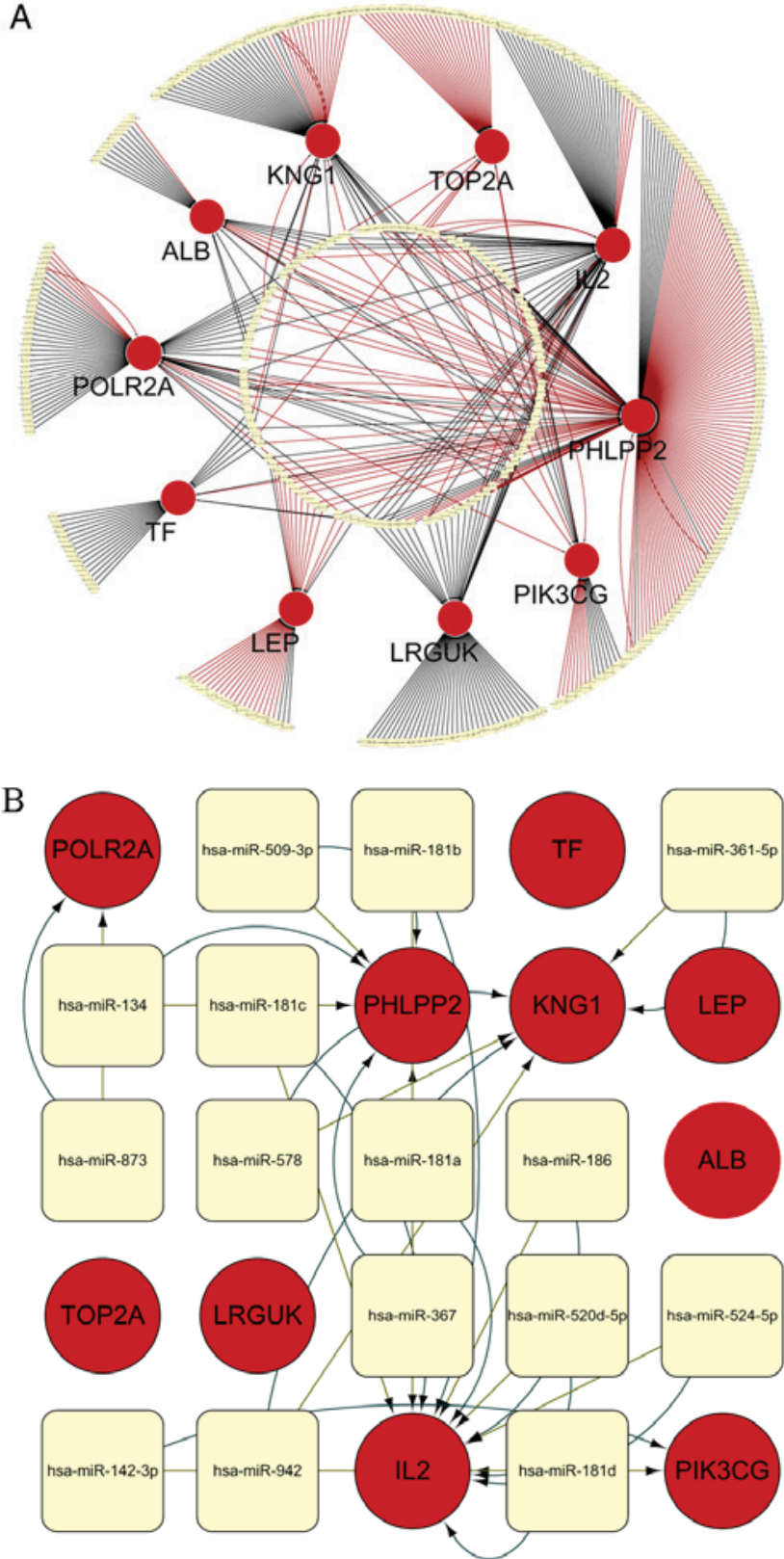

Figure 4. miRNA regulation of genes. (A) Extended regulatory network of miRNAs and target genes. The MTIs obtained from the MicroCosm database $(n=305)$ and TargetScan database $(n=277)$ are indicated in black and red, respectively. The overlap threshold function was set to display only MTIs present in 2 regulatory interaction networks. (B) Defined number of predicted miRNAs and target genes. Genes and miRNAs are denoted as red circles and yellow rounded rectangles, respectively. Additionally, green edges represent MTIs from the database TargetScan v6.2, and yellow edges represent MTIs from the database MicroCosm v5.0. The names of the genes and miRNAs are displayed on the nodes. miRNA, microRNA; MTI, microRNA-target interaction.

$A L B$ had the highest degree of connectivity. These hub genes, particularly $A L B, T O P 2 A, K N G 1, I L 2, P I K 3 C G, L E P$ and $T F$, were enriched for homeostatic processes and the regulation of apoptotic processes. A recent study has reported that homeostatic processes are imbalanced in postmenopausal osteoporotic women (25). In addition, triggering apoptotic processes could promote osteoclast apoptosis, thus alleviating OP (26). It could be hypothesized that the hub genes are associated with the development of OP. $A L B$ and its product, 
Table VI. A list of 5 hub genes and their predicted miRNAs from the CyTargetLinker extension network analysis ${ }^{\mathrm{a}}$.

\begin{tabular}{|c|c|}
\hline Gene & miRNAs \\
\hline IL2 & $\begin{array}{l}\text { hsa-miR-524-5p, hsa-miR-520d-5p, } \\
\text { hsa-miR-181b, hsa-miR-181a,hsa-miR-181d, } \\
\text { hsa-miR-181c, hsa-miR-186 }\end{array}$ \\
\hline PHLPP2 & hsa-miR-509-3p, hsa-miR-134, hsa-miR-367 \\
\hline KNG1 & hsa-miR-361-5p, hsa-miR-578, hsa-miR-942 \\
\hline PIK3CG & hsa-miR-142-3p \\
\hline POLR2A & hsa-miR-873 \\
\hline
\end{tabular}

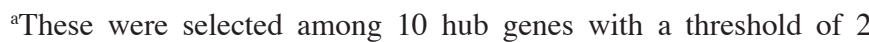
regulatory interaction networks. The miRNA-target interactions were obtained from the MicroCosm database (305) and TargetScan database (277). miRNA/miR, microRNA; IL2, interleukin 2; PHLPP2, $\mathrm{PH}$ domain leucine-rich repeat-containing protein phosphatase 2; KNG1, kininogen 1; PIK3CG, phosphatidylinositol-4,5-bisphosphate 3-kinase catalytic subunit $\gamma$; POLR2A, RNA polymerase II subunit A.

which has a good binding capacity for hormones, is involved in various BPs, including antioxidant activity, the regulation of apoptotic processes and the cellular response to starvation. The precise function of $A L B$ and its role in OP are unclear. However, a previous study found that the expression of ALB differed significantly between a normal group and an OP group $(\mathrm{P}<0.05)$, and others have linked oxidative status with bone alterations (27-29). PHLPP2 is involved in the regulation of protein kinase $\mathrm{B}$ (Akt) and protein kinase $\mathrm{C}$ signaling, and mediates dephosphorylation in the $\mathrm{C}$-terminal domain hydrophobic motif of members of the AGC serine/threonine protein kinase family. The protein is also involved in signal transduction and hippocampus development. PHLPP2 exhibits nuclear localization and is expressed in numerous tissue types, including the small intestine, brain, bone marrow and ovaries (30). PHLPP inhibitors may relieve mechanical pain and slow cartilage degradation in osteoarthritic joints (31). Therefore, $P H L P P 2$ has a potential regulatory role in OP and is a target for future research. $T O P 2 A$, which encodes a DNA topoisomerase, is associated with various processes, including chromosome condensation (32). Mutations in TOP $2 \mathrm{~A}$ are associated with the development of drug resistance, including etoposide and doxorubicin (33-35). Exposure to atmospheric oxygen promotes TOP2A expression in mouse MSCs, leading to oxidative stress, reduced cell viability and the inhibition of cell proliferation (36). KNGI exhibits alternative splicing to produce 2 distinct proteins: High- and low-molecular-weight kininogen. $K N G 1$ also factors in the G-protein-coupled receptor signaling pathway and the inflammatory response. Zhang et al (37) revealed that KNG1 is significantly associated with the complement and coagulation cascade pathway in Kashin-Beck disease. Further research is required to clarify the role of $K N G 1$ in OP. IL2 proteins are involved in cytokine secretion and are vital for the proliferation of $\mathrm{T}$ and B lymphocytes. Targeted knock-outs of similar genes in mice cause ulcerative diseases, suggesting that IL2 serves an important role in antigen-stimulated immune responses (38). The present study revealed that $I L 2$ is associated with the positive regulation of tissue remodeling, including bone remodeling, but little is known about the effects and mechanisms of IL2 in OP. LRGUK comprises 3 domains: A leucine-rich repeat, a guanylate kinase-like domain and an unnamed domain (39). LRGUK exhibits biased expression in the testis, bone marrow and lungs, and functions in ATP-binding and kinase activity. Studies on $L R G U K$ are rare. One previous study demonstrated that $L R G U K$ is associated with type 2 diabetes and fasting glucose levels (40). PIK3CG phosphorylates inositol lipids and is involved in the immune response process; it serves a vital role by recruiting pleckstrin homology domain-containing proteins to the membrane, including Akt1, and by activating signaling cascades involved in cell growth, proliferation and differentiation (41). Additionally, PIK3CG regulates bone homeostasis by modulating osteoclastogenesis and bone homeostasis (42), suggesting that this gene is linked to OP. LEP encodes a protein secreted by white adipocytes and serves an important role in energy balance regulation. This protein is also involved in endocrine function and in the regulation of immune and inflammatory responses. Tariq et al (43) found that body weight and body mass index impact BMD, whereas serum leptin is not associated with BMD. Zheng et al (44) identified that LEP overexpression in bone marrow stromal cells can stimulate periodontal regeneration in osteoporotic conditions. The association between $L E P$ and $\mathrm{OP}$ is unclear, and should be a focus of future studies. $T F$ encodes an extracellular or secreted glycoprotein with an $\mathrm{N}$-terminal transmembrane domain and a short cysteine-rich cytoplasmic loop prior to the unique C-terminal ends (45). The protein is involved in cellular iron ion homeostasis, post-translational protein modification and the regulation of protein stability. The putative iron sensor TF receptor 2 can bind to iron-loaded TF in the bloodstream, which increases the expression of hepcidin by stimulating the bone morphogenetic protein signaling pathway (46). POLR2A provides a platform for transcription, mRNA processing and chromosome remodeling (47). This gene encodes an essential subunit of RNA polymerase II and is ubiquitously expressed in the testis, skin and bone marrow, and is involved in numerous physiological processes, including the regulation of gene silencing by miRNA and of RNA splicing $(48,49)$. However, the biological functions of $P O L R 2 A$ in OP remain unknown.

A module analysis of the PPI network demonstrated that OP is linked to neuroactive ligand-receptor interactions and ubiquitin-mediated proteolysis. The neuroactive ligand-receptor interaction pathway can be activated by targeting the cell membrane $\mathrm{G}$ protein coupled receptor, which is involved in signal transduction from the extracellular to the intracellular components, including glycine, serine and threonine $(50,51)$. Neuroactive steroids, which influence the modulation of the $\gamma$-aminobutyric acid receptor, are hormones that act as regulators of neurotransmitter receptors to either enhance or suppress neuronal activity (52). Moreover, ubiquitin-mediated proteolysis has been reported to serve a key role in MSC osteogenic or adipogenic differentiation (53) and the ubiquitin-mediated proteolysis pathway serves a critical role in various processes, including the cell cycle, cellular response to stress, DNA repair and immune regulation (54). Therefore, combined with the present results, these data indicate that neuroactive 
ligand-receptor interactions and ubiquitin-mediated proteolysis may serve key roles in the progression of OP, and are potential targets for drug development and treatment.

In the present study, an integrated miRNA-gene analysis of core genes associated with OP was performed. An overlap threshold of 2 was set to identify only interactions supported by 2 MTI databases, and to ensure credible and accurate results. Overall, 15 predicted miRNAs and their target genes were screened. IL2 was regulated by miRNAs hsa-miR-524-5p, hsa-miR-520d-5p, hsa-miR-181b, hsa-miR-181a, hsa-miR-181d, hsa-miR-181c and hsa-miR-186. These miRNAs were enriched for biological function terms, including 'molecular function', 'enzyme regulator activity' and 'immune system process'. PHLPP2 was associated with miRNAs hsa-miR-509-3p, hsa-miR-134 and hsa-miR-367, which are mainly associated with organelles. miRNA hsa-miR-361-5p was observed to modulate KNG1, along with hsa-miR-942 and hsa-miR-578. These are involved in blood coagulation and platelet activation. Furthermore, hsa-miR-142-3p may serve a vital role in OP by regulating $P I K 3 C G$, which is involved in the estrogen and $\mathrm{B}$ cell receptor signaling pathways. miRNA hsa-miR-873, which is associated with metabolic pathways, co-regulates $P O L R 2 A$. The present results indicate that a series of miRNAs simultaneously regulate the same gene, as observed for IL2, PHLPP2 and $K N G 1$. Further studies are required to verify these interactions.

In conclusion, the results of the present study improve our understanding of the progression of OP based on an in-depth bioinformatics analysis of DEGs. In total, 915 DEGs and 10 core genes (ALB, PHLPP2, TOP2A, KNG1,IL2, LRGUK, $P I K 3 C G, L E P, T F$ and POLR2A) involved in OP were identified. Additionally, miRNAs associated with target genes were identified; these may be crucial for the initiation and progression of OP. However, further experimental studies are required in order to determine the precise roles of these genes and miRNAs. The present study provides a useful set of genes and miRNAs for future studies on the molecular mechanisms of OP and for the determination of therapeutic targets.

\section{Acknowledgements}

Not applicable.

\section{Funding}

The National Natural Science Foundation of China (grant no. 81573874) provided financial support for the conduct of the study.

\section{Availability of data and materials}

The datasets generated and analyzed during the current study are available in the Gene Expression Omnibus (GEO) repository (https://www.ncbi.nlm.nih.gov/geo/) (8).

\section{Authors' contributions}

QLF, YC and FT designed the study. YC, SMY, FT and FXL analyzed and interpreted the data. YC, FT and QLF were major contributors in the writing of the manuscript. All authors read and approved the submitted manuscript.

\section{Ethics approval and consent to participate}

Not applicable.

\section{Patient consent for publication}

Not applicable.

\section{Competing interests}

The authors declare that they have no competing interests.

\section{References}

1. Body JJ, Bergmann P, Boonen S, Boutsen Y, Devogelaer JP, Goemaere S, Kaufman JM, Rozenberg S and Reginster JY: Evidence-based guidelines for the pharmacological treatment of postmenopausal osteoporosis: A consensus document by the Belgian Bone Club. Osteoporos Int 21: 1657-1680, 2010.

2. Chen P, Li Z and Hu Y: Prevalence of osteoporosis in China: A meta-analysis and systematic review. BMC Public Health 16: 1039,2016

3. Svedbom A, Hernlund E, Ivergård M, Compston J, Cooper C, Stenmark J, McCloskey E, Jönsson B and Kanis J; EU Review Panel of IOF: Osteoporosis in the European Union: A compendium of country-specific reports. Arch Osteoporos 8: 137, 2013.

4. Ma M, Chen X, Lu L, Yuan F, Zeng W, Luo S, Yin F and Cai J: Identification of crucial genes related to postmenopausal osteoporosis using gene expression profiling. Aging Clin Exp Res 28: 1067-1074, 2016

5. Takayanagi H: Osteoimmunology: Shared mechanisms and crosstalk between the immune and bone systems. Nat Rev Immunol 7: 292-304, 2007.

6. Li X, Ge H, Weiszmann J, Hecht R, Li Y, Véniant M, Xu J, Wu X, Lindberg R and Li Y: Inhibition of lipolysis may contribute to the acute regulation of plasma FFA and glucose by FGF21 in ob/ob mice. FEBS Lett 583: 3230-3234, 2009.

7. Hao RH, Gao JL, Li M, Huang W, Zhu DL, Thynn HN, Dong SS and Guo Y: Association between fibroblast growth factor 21 and bone mineral density in adults. Endocrine 2018.

8. Barrett T, Wilhite SE, Ledoux P, Evangelista C, Kim IF, Tomashevsky M, Marshall KA, Phillippy KH, Sherman PM, Holko M, et al: NCBI GEO: Archive for functional genomics data sets-update. Nucleic Acids Res 41 (Database Issue): D991-D995, 2012.

9. Kanehisa M and Goto S: KEGG: Kyoto encyclopedia of genes and genomes. Nucleic Acids Res 28: 27-30, 2000.

10. Ashburner M, Ball CA, Blake JA, Botstein D, Butler H, Cherry JM, Davis AP, Dolinski K, Dwight SS, Eppig JT, et al: Gene ontology: Tool for the unification of biology. Nature Genet 25: 25-29, 2000.

11. Benisch P, Schilling T, Klein-Hitpass L, Frey S, Seefried L, Raaijmakers N, Krug M, Regensburger M, Zeck S, Schinke T, et al: The transcriptional profile of mesenchymal stem cell populations in primary osteoporosis is distinct and shows overexpression of osteogenic inhibitors. PLoS One 7: e45142, 2012.

12. Venet D, Detours V and Bersini H: A measure of the signal-to-noise ratio of microarray samples and studies using gene correlations. PLoS One 7: e51013, 2012.

13. Huang da W, Sherman BT and Lempicki RA: Systematic and integrative analysis of large gene lists using DAVID bioinformatics resources. Nat Protoc 4: 44-57, 2009.

14. Bindea G, Mlecnik B, Hackl H, Charoentong P, Tosolini M, Kirilovsky A, Fridman WH, Pagès F, Trajanoski Z and Galon J: ClueGO: A Cytoscape plug-in to decipher functionally grouped gene ontology and pathway annotation networks. Bioinformatics 25: 1091-1093, 2009.

15. Doncheva N, Assenov Y, Domingues F and Albrecht $M$ : Topological analysis and interactive visualization of biological networks and protein structures. Nat Protoc 7: 670-685, 2012.

16. Szklarczyk D, Franceschini A, Wyder S, Forslund K, Heller D, Huerta-Cepas J, Simonovic M, Roth A, Santos A, Tsafou KP, et al: STRING v10: Protein-protein interaction networks, integrated over the tree of life. Nucleic Acids Res 43 (Database Issue): D447-D452, 2015. 
17. Shannon P, Markiel A, Ozier O, Baliga NS, Wang JT, Ramage D, Amin N, Schwikowski B and Ideker T: Cytoscape: A software environment for integrated models of biomolecular interaction networks. Genome Res 13: 2498-2504, 2003.

18. Bader GD and Hogue CW: An automated method for finding molecular complexes in large protein interaction networks. BMC Bioinformatics 4: 2, 2003.

19. Benjamini Y and Hochberg Y: Controlling the false discovery rate: A practical and powerful approach to multiple testing. J Roy Stat Soc Ser B 57: 289-300, 1995.

20. Kutmon M, Kelder T, Mandaviya P, Evelo CT and Coort SL: CyTargetLinker: A Cytoscape app to integrate regulatory interactions in network analysis. PLoS One 8: e82160, 2013.

21. Tella SH and Gallagher JC: Prevention and treatment of postmenopausal osteoporosis. J Steroid Biochem Mol Biol 142: $155-170,2014$

22. Ho SS, Vollmer NL, Refaat MI, Jeon O, Alsberg E, Lee MA and Leach JK: Bone morphogenetic protein-2 promotes human mesenchymal stem cell survival and resultant bone formation when entrapped in photocrosslinked alginate hydrogels. Adv Healthc Mater 5: 2501-2509, 2016.

23. Maslov $\mathrm{S}$ and Sneppen K: Specificity and stability in topology of protein networks. Science 296: 910-913, 2002.

24. Wu B, Xie J, Du Z, Wu J, Zhang P, Xu L and Li E: PPI network analysis of mRNA expression profile of ezrin knockdown in esophageal squamous cell carcinoma. Biomed Res Int 2014: 651954, 2014.

25. Korkmaz V, Kurdoglu Z, Alisik M, Turgut E, Sezgın OO, Korkmaz H, Ergun Y and Erel O: Thiol/disulfide homeostasis in postmenopausal osteoporosis. J Endocrinol Invest 40: 431-435, 2017

26. Lee EJ, Kim JL, Kim YH, Kang MK, Gong JH and Kang YH: Phloretin promotes osteoclast apoptosis in murine macrophages and inhibits estrogen deficiency-induced osteoporosis in mice. Phytomedicine 21: 1208-1215, 2014.

27. Huang C and Li S: Association of blood neutrophil lymphocyte ratio in the patients with postmenopausal osteoporosis. Pak J Med Sci 32: 762-765, 2016.

28. Spilmont M, Léotoing L, Davicco MJ, Lebecque P, Mercier S, Miot-Noirault E, Pilet P, Rios L, Wittrant Y and Coxam V: Pomegranate and its derivatives can improve bone health through decreased inflammation and oxidative stress in an animal model of postmenopausal osteoporosis. Eur J Nutr 53: 1155-1164, 2014

29. Wauquier F, Leotoing L, Coxam V, Guicheux J and Wittrant $Y$ : Oxidative stress in bone remodelling and disease. Trends Mol Med 15: 468-477, 2009.

30. Fagerberg L, Hallström BM, Oksvold P, Kampf C, Djureinovic D, Odeberg J, Habuka M, Tahmasebpoor S, Danielsson A, Edlund K, et al: Analysis of the human tissue-specific expression by genome-wide integration of transcriptomics and antibody-based proteomics. Mol Cell Proteomics 13: 397-406, 2014.

31. Hwang SM, Feigenson M, Begun DL, Shull LC, Culley KL, Otero M, Goldring MB, Ta LE, Kakar S, Bradley EW and Westendorf JJ Phlpp inhibitors block pain and cartilage degradation associated with osteoarthritis. J Orthop Res 36: 1487-1497, 2018.

32. Madabhushi R: The Roles of DNA Topoisomerase II $\beta$ in Transcription. Int J Mol Sci 19: pii: E1917, 2018

33. Lang AJ, Mirski SE, Cummings HJ, Yu Q, Gerlach JH and Cole SP: Structural organization of the human TOP2A and TOP2B genes. Gene 221: 255-266, 1998.

34. Kaplan E and Gündüz U: Expression analysis of TOP2A, MSH2 and MLH1 genes in MCF7 cells at different levels of etoposide resistance. Biomed Pharmacother 66: 29-35, 2012.

35. Raguz S, Adams C, Masrour N, Rasul S, Papoutsoglou P, Hu Y, Cazzanelli G, Zhou Y, Patel N, Coombes C, et al: Loss of $\mathrm{O}^{6}$-methylguanine-DNA methyltransferase confers collateral sensitivity to carmustine in topoisomerase II-mediated doxorubicin resistant triple negative breast cancer cells. Biochem Pharmacol 85: 186-196, 2013.

36. Boregowda SV, Krishnappa V, Chambers JW, Lograsso PV, Lai WT, Ortiz LA and Phinney DG: Atmospheric oxygen inhibits growth and differentiation of marrow-derived mouse mesenchymal stem cells via a p53-dependent mechanism: Implications for long-term culture expansion. Stem Cells 30 : 975-987, 2012
37. Zhang F, Wen Y, Guo X, Zhang Y, Wang S, Yang T, Shen H, Chen X, Tan L, Tian Q and Deng HW: Genome-wide pathway-based association study implicates complement system in the development of Kashin-Beck disease in Han Chinese. Bone 71: 36-41, 2015.

38. Li J, Hou Y, Ding X, Hou W, Song B and Zeng Y: Overexpression, purification, molecular characterization and the effect on tumor growth of ribosomal protein L22 from the Giant Panda (Ailuropoda melanoleuca). Mol Biol Rep 41: 3529-3539, 2014.

39. Okuda H, DeBoer K, O'Connor AE, Merriner DJ, Jamsai D and O'Bryan MK: LRGUK1 is part of a multiprotein complex required for manchette function and male fertility. FASEB J 31: 1141-1152, 2017.

40. Laramie JM, Wilk JB, Williamson SL, Nagle MW, Latourelle JC, Tobin JE, Province MA, Borecki IB and Myers RH: Polymorphisms near EXOC4 and LRGUK on chromosome $7 \mathrm{q} 32$ are associated with Type 2 Diabetes and fasting glucose; the NHLBI Family Heart Study. BMC Med Genet 9: 46,2008 .

41. Jean S and Kiger AA: Classes of phosphoinositide 3-kinases at a glance. J Cell Sci 127: 923-928, 2014.

42. Kang H, Chang W, Hurley M, Vignery A and Wu D: Important roles of PI3Kgamma in osteoclastogenesis and bone homeostasis. Proc Natl Acad Sci USA 107: 12901-12906, 2010.

43. Tariq S, Baig M, Tariq S and Shahzad M: Association of serum leptin with bone mineral density in postmenopausal osteoporotic females. Gynecol Endocrinol 33: 287-291, 2017.

44. Zheng B, Jiang J, Chen Y, Lin M, Du Z, Xiao Y, Luo K and Yan F: Leptin overexpression in bone marrow stromal cells promotes periodontal regeneration in a rat model of osteoporosis. J Periodontol 88: 808-818, 2017.

45. Ramsey J and Mukhopadhyay S: Disentangling the frames, the state of research on the alphavirus $6 \mathrm{~K}$ and $\mathrm{TF}$ proteins. Viruses 9: pii: E228, 2017.

46. Worthen $\mathrm{C}$ and Enns C: The role of hepatic transferrin receptor 2 in the regulation of iron homeostasis in the body. Front Pharmacol 5: 34, 2014.

47. Zhao DY, Gish G, Braunschweig U, Li Y, Ni Z, Schmitges FW, Zhong G, Liu K, Li W, Moffat J, et al: SMN and symmetric arginine dimethylation of RNA polymerase II C-terminal domain control termination. Nature 529: 48-53, 2016.

48. Hui-Ying X, Da-Hong Z, Li-Juan J and Xiao-Jie L: Anticancer opportunity created by loss of tumor suppressor genes. Technol Cancer Res Treat 15: 729-731, 2016.

49. David CJ, Boyne AR, Millhouse SR and Manley JL: The RNA polymerase II C-terminal domain promotes splicing activation through recruitment of a U2AF65-Prp19 complex. Genes Dev 25: 972-983, 2011.

50. Lauss $M$, Kriegner A, Vierlinger $K$ and Noehammer $C$ : Characterization of the drugged human genome. Pharmacogenomics 8: 1063-1073, 2007.

51. Bhattarai UR, Katuwal Bhattarai M, Li F and Wang D: Insights into the temporal gene expression pattern in lymantria dispar larvae during the baculovirus induced hyperactive stage. Virol Sin: Jul 25, 2018 (Epub ahead of print).

52. Wang J, Cheng J, Zhang C and Li X: Cardioprotection effects of sevoflurane by regulating the pathway of neuroactive ligand-receptor interaction in patients undergoing coronary artery bypass graft surgery. Comput Math Methods Med 2017: 3618213, 2017.

53. Zhuang Q, Mao W, Xu P, Li H, Sun Z, Li S, Qiu G, Li J and Zhang J: Identification of differential genes expression profiles and pathways of bone marrow mesenchymal stem cells of adolescent idiopathic scoliosis patients by microarray and integrated gene network analysis. Spine (Phila Pa 1976) 41: 840-855, 2016.

54. Ciechanover A, Orian A and Schwartz AL: Ubiquitin-mediated proteolysis: Biological regulation via destruction. Bioessays 22: 442-451, 2000 